\title{
Contribution of Sentinel-2/Landsat-8 OLI Images to Extracting Vegetation Cover and Wetlands Area in Urban Zones: Case of the Dakar Region (Senegal)
}

\author{
Dome Tine', Gayane Faye², Omar Marico², Modou Mbaye², Landing Biaye², Mbagnick Faye ${ }^{3}$, \\ Guilgane Faye ${ }^{3}$
}

${ }^{1}$ Département de Géographie, Chercheur Associé au Laboratoire de Télédétection Appliquée (LTA), Institut des Sciences de la Terre (IST), Université Cheikh Anta DIOP de Dakar, Dakar, Sénégal

${ }^{2}$ Laboratoire de Télédétection Appliquée (LTA), Institut des Sciences de la Terre (IST), Université Cheikh Anta DIOP de

Dakar, Dakar, Sénégal

${ }^{3}$ Département de Géographie, Cheikh Anta DIOP University of Dakar, Dakar, Sénégal

Email: dometine85@gmail.com, gayane.faye@yahoo.fr

How to cite this paper: Tine, D., Faye, G., Marico, O., Mbaye, M., Biaye, L., Faye, M. and Faye, G. (2021) Contribution of Sentinel-2/Landsat-8 OLI Images to Extracting Vegetation Cover and Wetlands Area in Urban Zones: Case of the Dakar Region (Senegal). Journal of Geographic Information System, 13, 523-537. https://doi.org/10.4236/jgis.2021.134029

Received: June 1, 2021

Accepted: August 8, 2021

Published: August 11, 2021

Copyright ( 2021 by author(s) and Scientific Research Publishing Inc. This work is licensed under the Creative Commons Attribution International License (CC BY 4.0).

http://creativecommons.org/licenses/by/4.0/ (c) (i) Open Access

\begin{abstract}
Urban areas house vegetation cover in several forms, fulfilling several ecological functions like thermal regulator, biodiversity, air quality, etc. However, their extent is often not very well known, especially in African cities, making it sometimes difficult to assess their real impact on the urban ecosystem functioning. This work aims to analyse the capacity of satellite sensors for mapping vegetation and wetlands in urban areas. The data produced by the MSI sensors of Sentinel 2 and OLI of Landsat- 8 are used to identify and map the vegetation cover in the Dakar region through a supervised classification with the Support Vector Machine (SVM) algorithm. The results show that it is sometimes not very easy to analyse urban vegetation with high spatial resolution images (HRS) resulting from the configuration of the vegetation in an urban environment, sometimes characterized by isolated trees or small green spaces. This explains why Sentinel-2 data which spatial resolution of 10 meters gives a better result compared to Landsat- 8 data which is 30 meters. However, a good rendering is noted for the vegetation around the wetlands area for the two sensors resulting from the high density and the size of the vegetated perimeters in this part of the capital. Overall, there is an underestimation of urban vegetation cover, particularly for Landsat-8. The use of very high spatial resolution images could be necessary to better assess the potential of satellite data for monitoring urban vegetation in Sahelian context.
\end{abstract}




\section{Keywords}

Remote Sensing, Urban Area, Vegetation, Wetland Area, Dakar

\section{Introduction}

The issue of urban landscape management has always been a headache for authorities in charge of cities, especially large agglomerations. It should be noted that despite their importance, tree vegetation can lower the temperature in a street by $2^{\circ} \mathrm{C}$, which can fight against urban heat islands [1], the monitoring and management of green spaces in the greater agglomeration has still not been easy.

With the advent of remote sensing, the study of urban environments characteristics has spectacularly grown thanks to the many possibilities offered by geospatial data. Knowledge about the extent and components of land use is important for urban development monitoring and planning, risk assessment, disaster management, and natural resource management.

The use of remote sensing in management first appeared in the early 1970s, with images provided by the Multispectral Scanner (MSS) sensor, mounted onboard the Landsat series satellites (1,2 and 3). However, the 80-meter spatial resolution of these images constitutes a limitation, as it does not allow to have information in a certain level of detail [2] [3] [4]. Indeed, urban spaces are characterized by a great heterogeneity with small sizes elements, while the radiometric value of the pixel represents the average of the signals reflected by the different objects that compose it. This makes it difficult to have "pure" pixels at this scale $(80 \mathrm{~m})$, the information provided by the pixel being composite, the pixel becomes mixed or "mixel" [5] in other words. From the 1980s, the second generation of satellites was launch with high spatial resolution (HSR) sensors $(30 \mathrm{~m}$ ) such as Landsat (TM, ETM+) and the most recent OLI sensor on Landsat-8 launched in 2013. Finally, Sentinel-2's high spatial resolution (10 m) MSI sensor, which was lunch in 2015, enriches this panoply of HSR data. It currently provides freely accessible images. The rapid advance in remote sensing has made it possible to have new types of very high spatial resolution (VHSR) images such as SPOT, Quickbird, IKONOS, Pleiades etc. These sensors, which provide images with increasingly fine details (less than $5 \mathrm{~m}$ ), provide more details on urban areas. However, unlike high spatial resolution images (HRS) which are often FREE access, VHSR images are paid data, which limits their accessibility for many cities, especially in Africa.

The objective of this study is to analyse the capacity of open access data (Landsat-8 and Sentinel-2) which are at HSR, for mapping vegetation in urban areas with a case study of the Dakar agglomeration. In this city, the vegetation cover rarely occupies large areas except classified forests and the edges of wetlands area. Note that the discriminating capacity of the sensors being a function of the size, organization and density of urban objects, the spatial resolution used 
to discriminate the urban structure must be adapted to each type of city [4] [6].

\subsection{Ecosystem Functions of Urban Vegetation}

According to the Intergovernmental Panel on Climate Change (IPCC), eleven of the twelve hottest years have been recorded since 1995 and would be attributable to the increased presence of greenhouse gases in the atmosphere. The reports The IPCC [7] [8] indicate an increase of the average temperatures in the coming decades. This climate change combined with the gradual loss of plant cover in urban areas will accentuate the heat island effect in cities [9]. However, urban vegetation would be an alternative solution given the many ecosystem services it offers. It improves the quality of life of city by reducing temperatures by intercepting solar radiation. Its presence in an urban environment would reduce the greenhouse gas emissions such as $\mathrm{CO}_{2}, \mathrm{~N}_{2} \mathrm{O}, \mathrm{CH}_{4}$ etc. The vegetation in its various configurations plays a role of cooler during high temperatures and of pollution filter (Figure 1). It can also be very useful in the management of storm water in urban areas. Leafy trees slow runoff by catching rain before the ground. Green roofs promote the temporary storage of rainwater and evapotranspiration [10].

Carbon sequestration by vegetation and soils helps reduce the $\mathrm{CO}_{2}$ content of the atmosphere [12]. Leaf areas such as the classified forest of Mbao and the surroundings of water points in the Dakar region, influence air quality. In a context of increased pollution to the point that Dakar is one of the most polluted cities in the world [13], vegetation intercepts polluting particles, absorbs some in gaseous form while producing oxygen. Its aerodynamic roughness modifies the winds and impacts the dispersion and deposition of polluting particles. From a geomorphological point of view, a strong vegetation cover protects the soil against runoff and the beating of rainwater. It fixes the soil and reduces water erosion. The presence of plant cover on the facades of buildings or inside houses (the most frequent case in Dakar) has an impact on the energy consumption of buildings. It intercepts solar radiation whose long near infrared wavelengths are strongly reflected and creates a shadow that softens temperatures.

Despite the important role that vegetation plays, the artificialization of urban

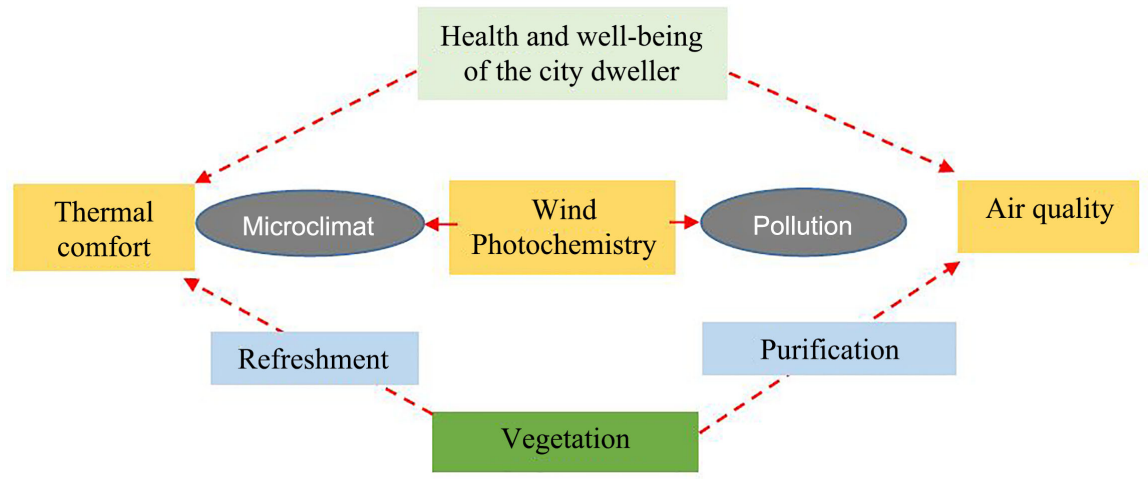

Figure 1. Microclimatic system and the role of vegetation in urban areas [11]. 
environments continues to make soils impermeable and increase the region's vulnerability to flooding. The Senegalese Promoville project is part of this dynamic. Accelerated artificialization which induces an increase in the speed of the flow, peak flows and the risk of flooding.

\subsection{General Framework}

The Dakar metropolitan area is located between $17^{\circ} 28^{\prime} 30^{\prime \prime}$ and $17^{\circ} 10^{\prime} 30^{\prime \prime} \mathrm{W}$ longitude and $14^{\circ} 33^{\prime \prime}$ and $14^{\circ} 51^{\prime \prime} \mathrm{N}$ latitude (Figure 2). It is located at the western end of the African continent, on a narrow peninsula. From an administrative point of view, the Dakar region is composite of four departments (Dakar, Guédiawaye, Pikine and Rufisque) and fifty-two (52) local communities. Temperatures vary between $17^{\circ} \mathrm{C}$ and $30^{\circ} \mathrm{C}$ depending on the season. Its maritime belt explains the fact that Dakar is characterized, during major period of the year, by a microclimate marked by the influence of the maritime trade winds [14]. Although the region belongs to the Sahelian domain, the climate of Dakar is of the Canarian type which is strongly influenced by geographic and atmospheric factors. Rainfall remains low at $537 \mathrm{~mm}$ per year. The geological formations of the Dakar region belong to the Senegalese-Mauritanian sedimentary basin and are characterized by Quaternary sandy accumulations which weld a rocky

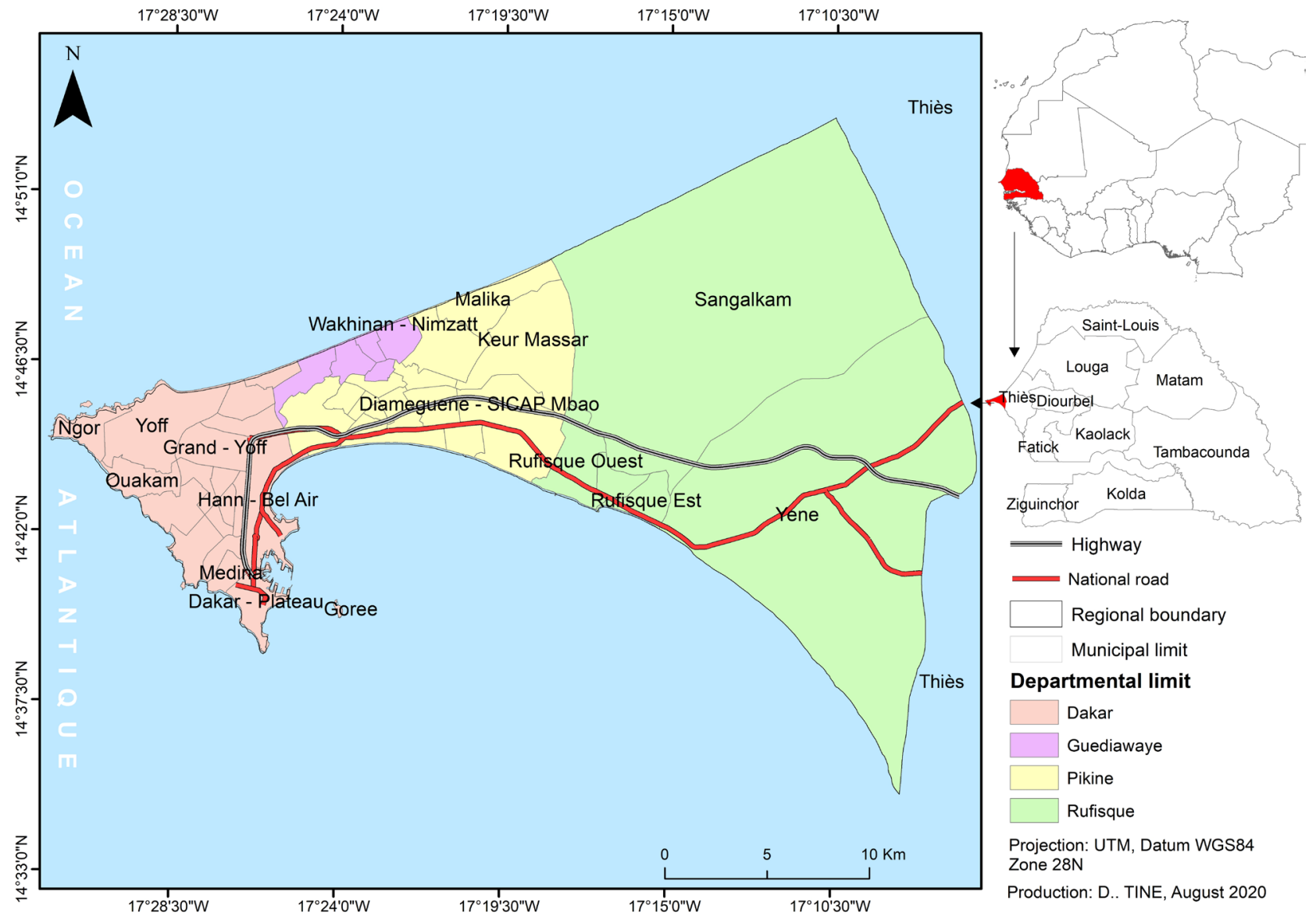

Figure 2. Geographical location of the Dakar region. 
head of volcanic origin and low plateaus shaped in marl and limestone, and adjoining the battleship massif of Dias [15]. From a geomorphological point of view, the region is marked by a flatness of its relief despite some slight topographical variations noted in the eastern part and to the west of the peninsula.

The vegetation cover of the Dakar region (Figure 2) is azonal due to the wide range of morpho-pedological landscapes and the moderate maritime climate. Two large groups stand out: anthropogenic vegetation resulting from human activities and composed of arboreal gardens, lawns, isolated trees, etc. and hygrophilic vegetation surrounding the wetlands.

\section{Materials and Methods}

\subsection{Data}

The Landsat-8 and Sentinel 2A images (Table 1) used are taken from the Google Earth Engine (GEE) platform, which is an infrastructure for archiving geospatial data in the cloud. It makes it possible to process and analyse large masses of satellite data (Big Data) online using an application programming interface (API) based on JavaScript or Python. Sentinel-2 and Landsat- 8 data are provided in apparent reflectance or at the top of the atmosphere (TOA) so they do not require radiometric calibration.

The images chosen are acquired in the non-rainy season, particularly between April and May in order to avoid any influence from the seasonal herbaceous vegetation that develops in the rainy season. The difference between the two types of images used lies in the spatial resolution and the number of spectral bands. The OLI radiometer acquires images in nine spectral bands ranging from visible

Table 1. Spectral and spatial characteristics of the satellite images used.

\begin{tabular}{|c|c|c|c|}
\hline Sensor & Acquisition date & Bands & Resolution \\
\hline \multirow{7}{*}{ MSI } & \multirow{7}{*}{ April-May } & 2-Blue & \multirow{3}{*}{$10 \mathrm{~m}$} \\
\hline & & 3-Green & \\
\hline & & 4-Red & \\
\hline & & 5-Vegetation classification & \multirow{3}{*}{$20 \mathrm{~m}$} \\
\hline & & 6-Vegetation classification & \\
\hline & & 7-Vegetation classification & \\
\hline & & 8-Near infrared & $10 \mathrm{~m}$ \\
\hline Sensor & Acquisition date & Bandes & Resolution \\
\hline \multirow{6}{*}{ OLI } & \multirow{6}{*}{ April-May } & 2-Blue & \multirow{6}{*}{$30 \mathrm{~m}$} \\
\hline & & 3-Green & \\
\hline & & 4-Red & \\
\hline & & 5-Near infrared & \\
\hline & & 6-Medium infrared 1 & \\
\hline & & 7-Medium infrared 2 & \\
\hline
\end{tabular}


to mid-infrared. While the Sentinel-2 MSI sensor takes images from visible to mid-infrared with different spatial resolutions (Table 1).

\subsection{Method of Detection and Extraction of Urban Vegetation}

The urban environment is a mixture of portions of roads, buildings, grassy areas, woods and bare soil. Their spectral reflectance can undergo significant variations according to their color, brightness, orientation or inclination [16]. Several factors influence the spectral signatures of objects in urban environment. The nature of the constructions, smooth surfaces, atmospheric pollution etc. can considerably modify the reflected of the signal. High spatial resolution remote sensing techniques make it possible to establish very precisely, with a small margin of error, the vegetation cover in urban areas [17].

In this study, the detection and extraction of information on satellite images is carried out on the one hand by pixel-oriented classification and on the other hand by spectral indices (Figure 3 ). In the first approach adopted relies on a supervised method of support vector machine (SVM) is used. It is an algorithm known for its efficiency in solving discrimination and regression problems and classifying complex data in order to produce good results. The protocol relies on user-supplied training areas that allow the algorithm to train there based on spectral responses of objects and classify the image.

The second approach consists in using the spectral indices of vegetation effective in the detection of the vegetation cover. Thus, the Normalized Difference Vegetation Index (NDVI) and the Adjusted Soil Vegetation Index (SAVI) were
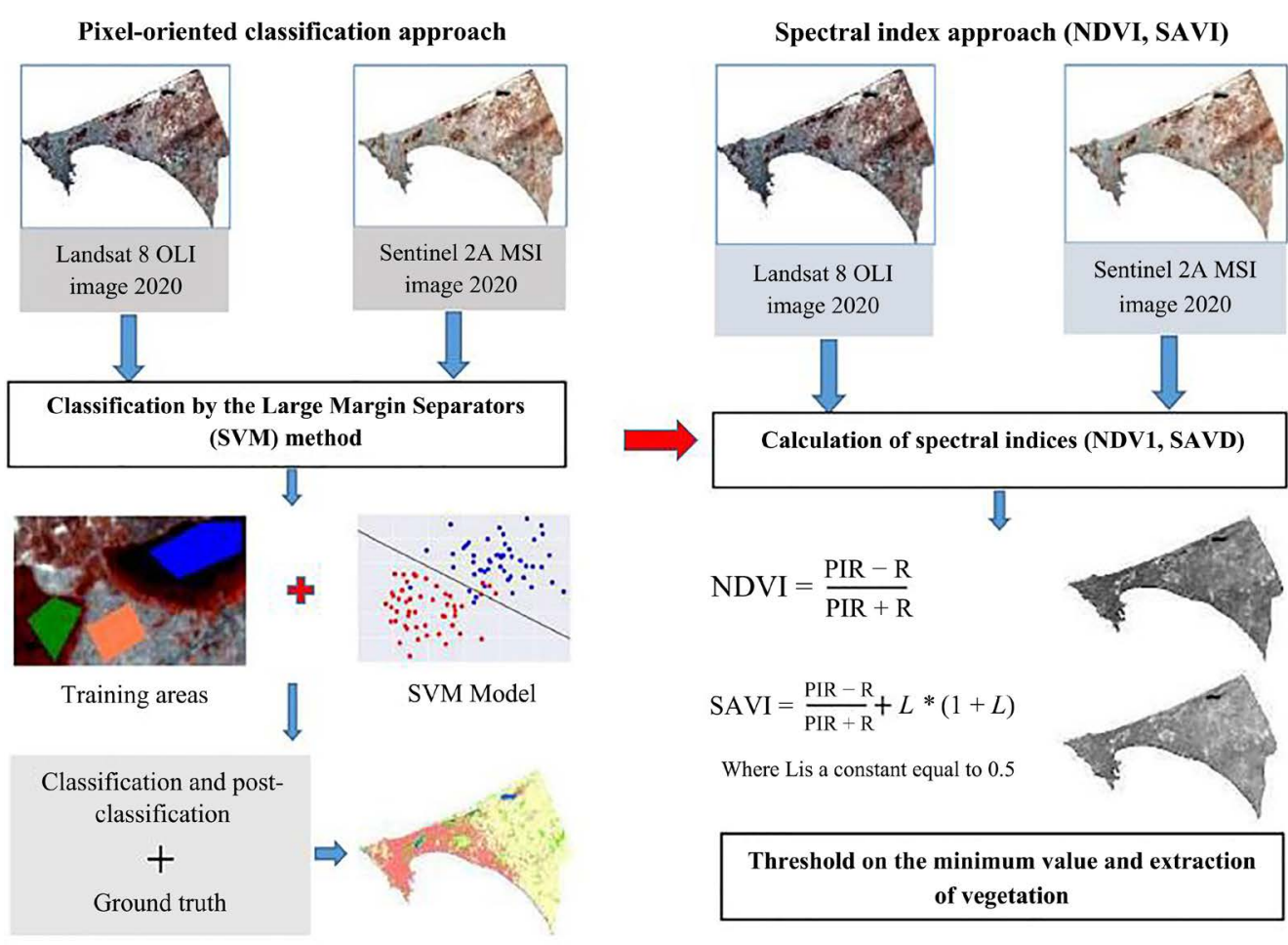

Figure 3. Vegetation extraction methodology in the Dakar region. 
applied to both images followed by thresholding on their minimum values. Thresholding is a direct operation applied to satellite images for decision making. This method presents a problem related to the estimation of the optimal threshold, which may vary depending on the images used. To fix the threshold value of the vegetation indices, a ground truth sample was used. Superimposing the coordinates of the sample with the multispectral image made it possible to retain an acceptable threshold. This method requires a priori knowledge of the reality on the ground.

According to the Ramsar Convention signed in 1971, wetlands area includes a great diversity of habitats: marshes, peat bogs, floodplains, rivers and lakes, coastal areas such as salt marshes, mangroves and river beds. The coral reefs and other marine areas not exceeding six meters in depth at low tide and artificial wetlands such as wastewater treatment ponds and retention lakes are also considered wetlands are. Wetlands are environments that strongly contribute to maintaining biodiversity and water quality [18]. They form considerable and diversified areas, the largest of which is in the Pikine department (Figure 4). They are also found in the north of the Dakar region and in Guediawaye and Rufisque. They are characterized by floodplain meadows found at the edge of wetlands area, characterized by periodic waterlogging, peatlands consisting mainly of an accumulation of organic matter and marine areas. Dakar's wetlands are increasingly threatened by urbanization while they constitute an important biodiversity conservation area [19]. Since a few years, wetlands area of the Dakar region has experienced strong demographic pressure linked to rampant urbanization.

Given to its heterogeneity (herbaceous, shrub and water formations), study of wetlands by spatial approach requires the potential of the data acquired at different scales. Each study scale corresponds to a level of observation and a specific tool [20]. However, the detection of wetlands therefore remains difficult from HSR data due to the similarities between different plant species, the low spatial resolution of the images and the low temporal repeatability [21]. This is the perspective of this study, which compares the contribution of high spatial resolution images from Sentinel-2 and landsat-8 OLI to the study of urban wetlands.
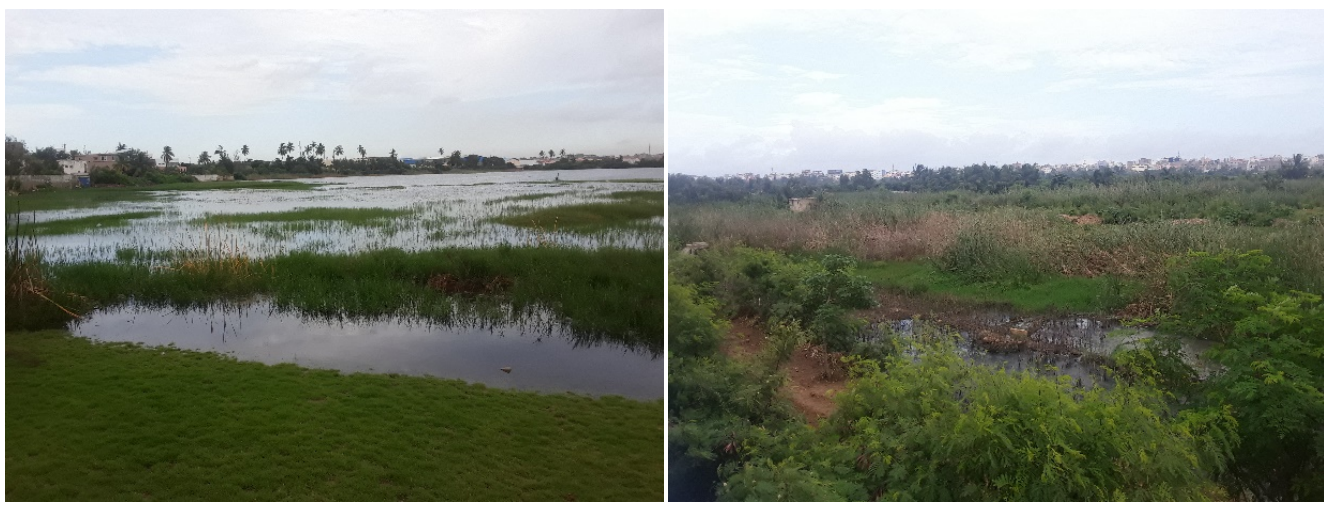

Figure 4. Sample of wetlands in the Dakar region: diversity and characteristics. 


\section{Results}

\subsection{Configuration of Urban Vegetation in the Dakar Region}

To study the vegetation cover in an urban environment by remote sensing, it is important to have an idea of their typology, shape and characteristics. Indeed, the urban landscape is very complex and presents a multifaceted configuration. In the Dakar region, vegetation is mainly composed of green facades, grassy surfaces, trees, arboreal gardens, green frames accompanying roads, car park fronts, cemeteries, wetlands and the surroundings water (Figure 5). Isolated trees, trees at the bottom of buildings, lianas around house fences cannot be highlighted due to the lighting conditions (buildings tend to create shade when the hour is passage of the satellite does not find the sun at the zenith). Homogeneous forms are rare, hence the complexity of identifying certain plant surfaces from satellite imagery.

\subsection{Analysis of the Spectral Signature of the Vegetation}

The identification and monitoring of plant cover is based on their reflectance according to the different wavelengths called the spectral signature. The variations in the spectral signature noted between the data from the two sensors are related to the difference in spatial resolution, the lighting conditions, the density and the configuration of the urban vegetation. The size of the pixel and the characteristic objects strongly influence the signal received by the sensor. A pixel of the Landsat-8 OLI image covers $900 \mathrm{~m}^{2}$, while a pixel of the Sentinel-2 image covers only $100 \mathrm{~m}^{2}$, in an urban environment with a high density of buildings,
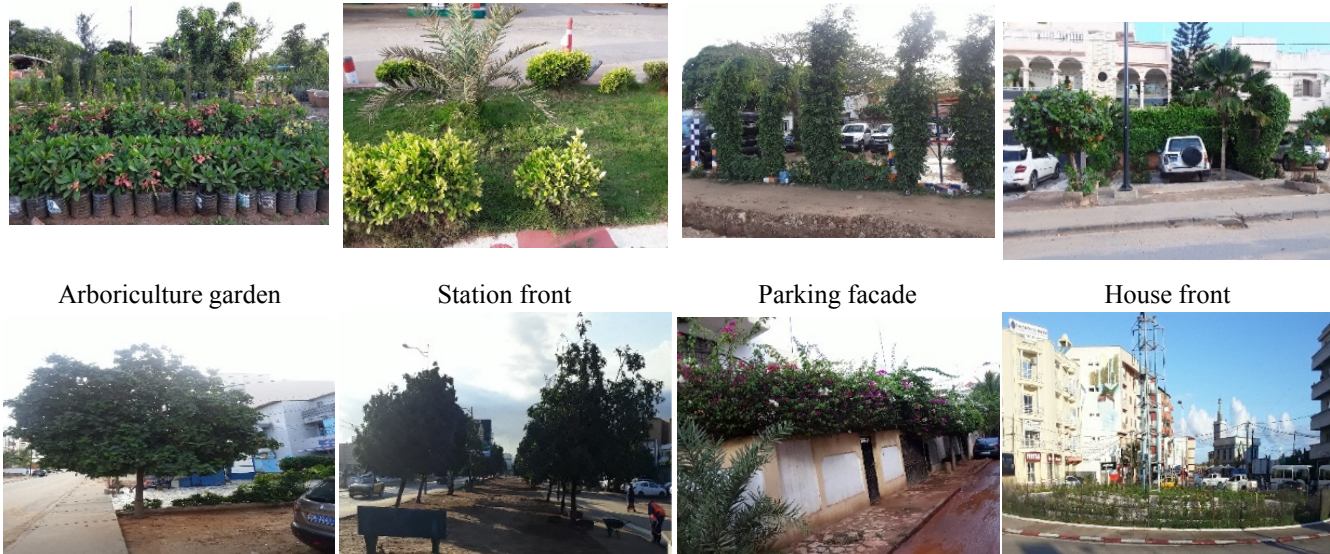

Isolated tree

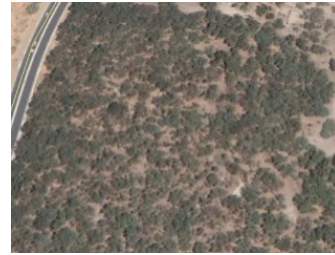

Mbao classified forest

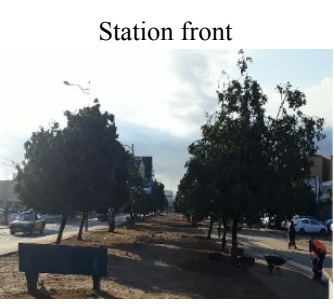

Trees along the roads

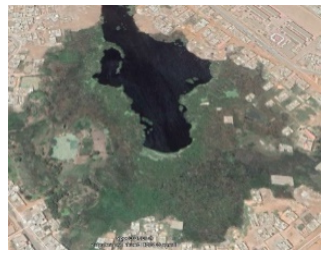

Water point

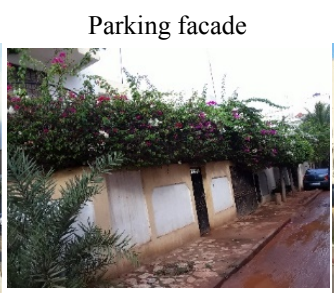

Lianas on the fences

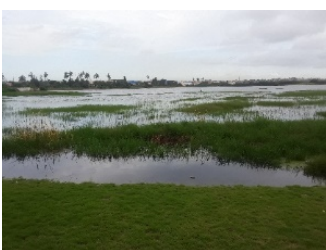

wetland
House front

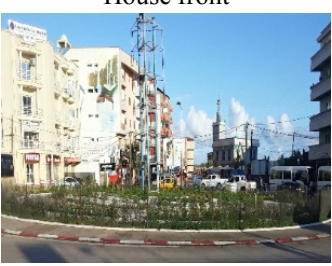

Roundabout

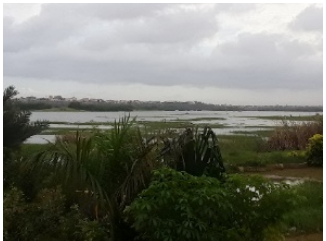

wetland

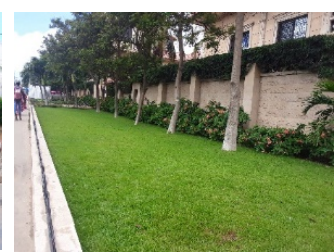

Lawn

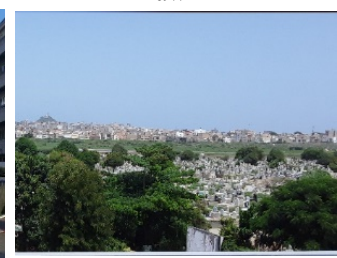

Cemeteries

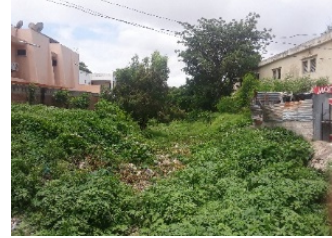

shallows

Figure 5. Characterisation of vegetation cover in the Dakar region. 
on an area of $900 \mathrm{~m}^{2}$ the possibility of having a mixture of objects (buildings, vegetation, bare ground) is high, which explains the spectral mixture of the pixels. This situation may be less with data from Sentinel-2. Analysis of Figure 6 reveals a strong reflectance of vegetation in bands 6, 7 and 8 of Sentinel-2. As everywhere else, the vegetation reflects strongly in the near infrared represented respectively by band 8 and band 5 of Sentinel- 2 and Landsat- 8 OLI.

However, certain variables can influence the spectral signature of the characteristic elements of the urban environment [22]. The disparity of the landscape leads to confusion of elements within a pixel and between pixels [6]. Therefore, the radiance value recorded for this pixel corresponds to a response including different ground cover [22]. Although vegetation reflects strongly in the near infrared, the mixing of the pixel can considerably attenuate the signal.

\subsection{Vegetation Extraction by Classification}

The SVM algorithm is applied to the two images to generate the land cover maps (Figure 7) of the studied region. The results were compared to the ground truth samples and to exogenous documents such as the topographic map of the region to produce the confusion matrix which allows to assess the quality of the classifications. It provides the overall details which provide information on the proportion of well-classified pixels and the Kappa index which gives an overall assessment of the quality of the information extraction. The literature considers that a classification is acceptable when the overall accuracies are greater than $60 \%$ and it is good between $70 \%$ and $80 \%$. However, both classifications can be considered reliable with respective overall accuracies of $96 \%$ and $88 \%$ for Sentinel $2 \mathrm{~A}$ and Landsat-8 OLI.

The qualitative analysis of the classification result (Figure 7) shows that the Sentinel-2 data gives much more precise results and estimates the vegetation at $7 \%$ of the land cover while with Landsat- 8 OLI, the vegetation occupies $5 \%$. This can be explained by the fineness of the spatial resolution of Sentinel-2, which makes it possible to detect smaller leaf areas in urban areas compared to Landsat- 8 OLI due to its coarse spatial resolution. Sentinel-2 vegetation pixel in dense built is found in mixed pixels with Landsat- 8 OLI, resulting in an underestimation of urban vegetation. However, the study showed that the OLI sensor is very sensitive to the reflectance of urban buildings providing more accurate results.
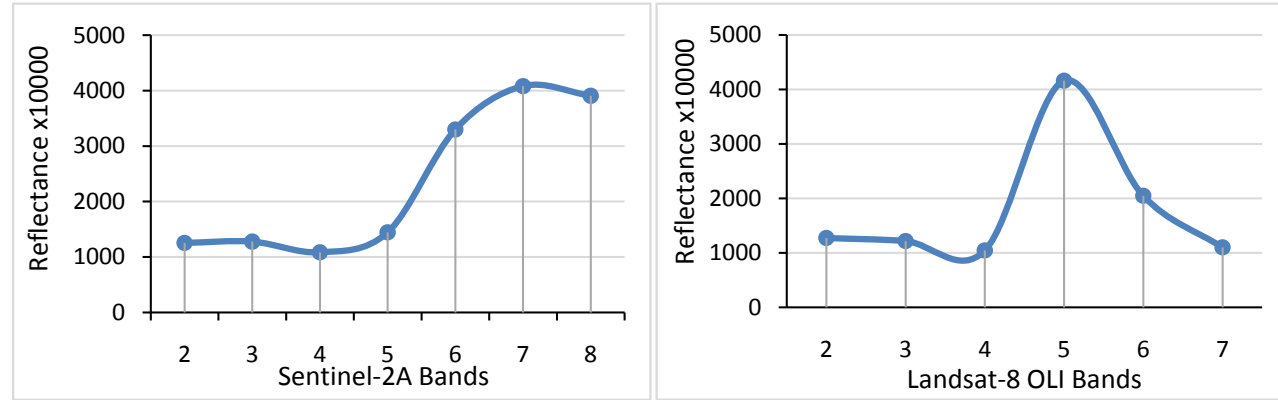

Figure 6. Spectral signature of urban vegetation as a function of OLI and MSI sensors. 

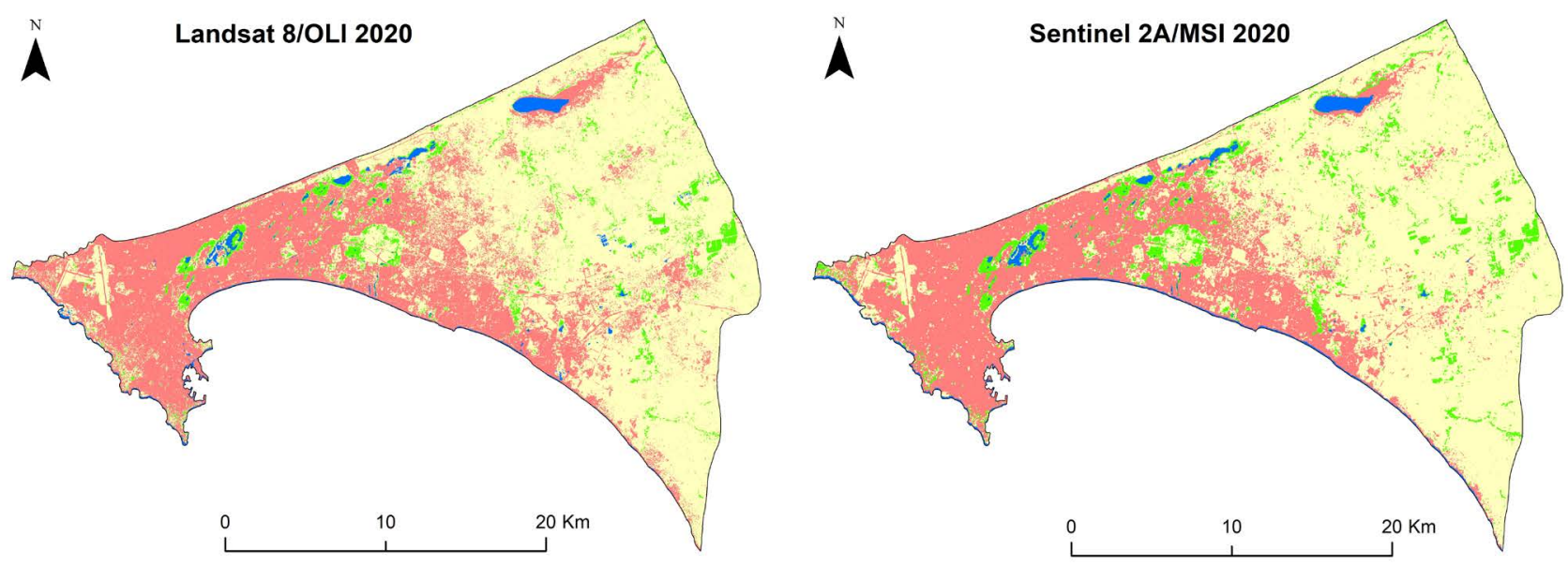

\begin{tabular}{|c|c|c|}
\hline Building & Vegetation & Water \\
\hline Classes & Sentinel 2A & Landsat 8 OLI \\
\hline Building & $30 \%$ & $34 \%$ \\
\hline Vegetation & $7 \%$ & $5 \%$ \\
\hline Water & $2 \%$ & $2 \%$ \\
\hline Bare soil & $61 \%$ & $59 \%$ \\
\hline
\end{tabular}

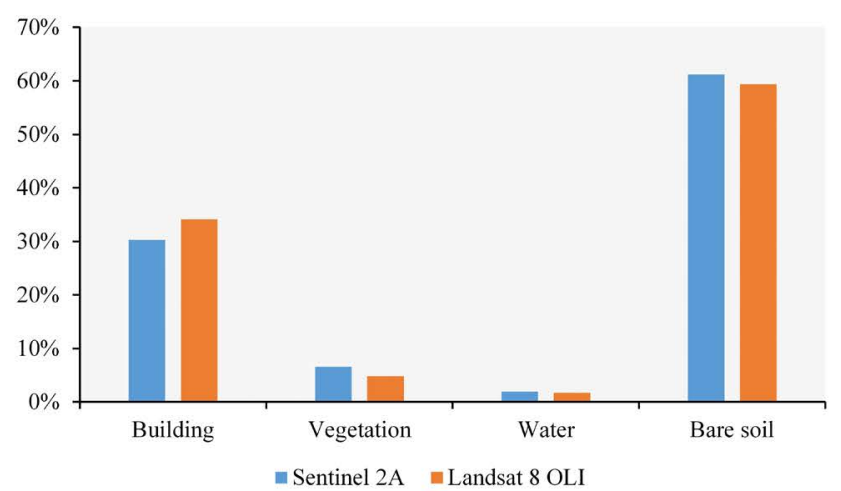

Figure 7. Land use by Sentinel 2A/MSI and Landsat-8/OLI between March and April 2020 in the Dakar region.

\subsection{Extraction by Vegetation Indices}

The threshold value is obtained after comparing the validation data and the vegetation indices. Thresholding is based on the minimum value of the vegetation index. The results provided by this method (Figure 8) show that the index approach is less precise than classifications. However, NDVI has limitations in complex area. In urban areas, for example, green spaces are characterized by a mixture of varying proportions of wooded areas, bare soil, built spaces, communication routes, etc. [23]. The spectral signature of these objects mixes with that of the vegetation cover, making the latter difficult to identify. This mix of pixels is not taken into account by the Normalized Difference Vegetation Index (NDVI) which is sensitive to the optical properties of the underlying soil, which can lead to significant errors if the vegetation that is being wished to characterize is not dense enough [24], as in urban areas. Results obtained from NDVI thresholding show the MSI sensor is more suitable for extracting urban vegetation. However, a second index, the SAVI (Soil Adjusted Vegetation Index), which takes into account the influence of soils, was calculated, taking into account the limits of NDVI. The signal reflected by the soils is separated from that of the plant cover in order to show more precisely the leaf surfaces. This index was applied to the images used and gave more precise results in terms of qualitative 

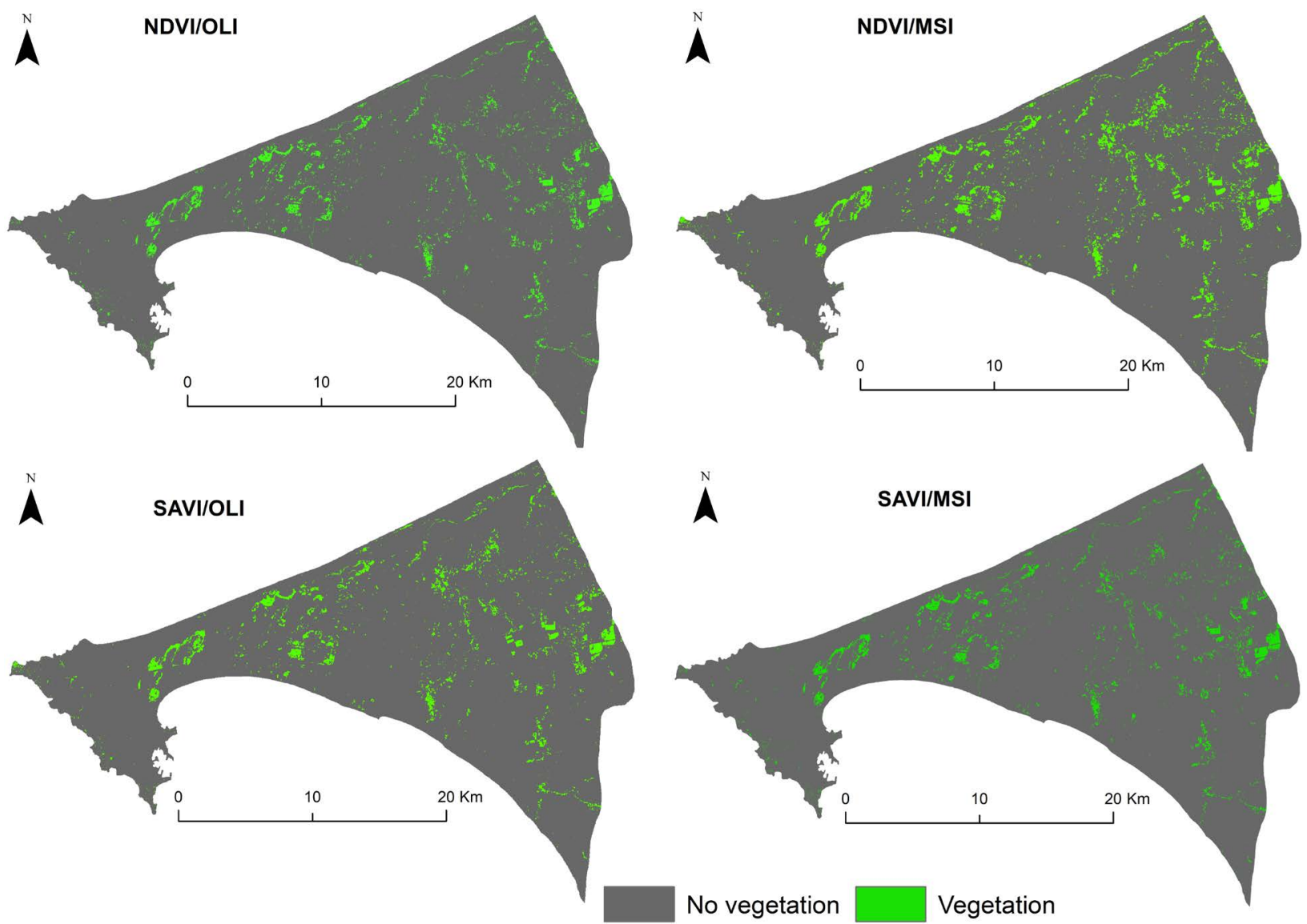

Figure 8. Extraction of urban vegetation by thresholding NDVI and SAVI in the Dakar region.

and quantitative analysis. The NDVI and the SAVI of Sentinel-2 MSI respectively estimate the urban vegetation at $6.69 \%$ and $4.74 \%$ of the total area of the studied space while the same indices calculated on the Landsat-8 OLI image estimate the plant cover at $4.42 \%$.

\subsection{Wetland Mapping}

Analysis of the classifications (Figure 9) showed sensitivity of the sensors to detecting wetlands. However, some errors appear due to the mix of pixels (water and vegetation) in this spatial resolution. The vegetation cover is in places extremely dense to the point that the water surface is undetectable by the sensor. These scenarios relate to small depressions (shallows and small areas of water conducive to the development of vegetation). The estimation of their areas is limited not only by the mix of pixels but also by their dynamic. The detection and identification of wetlands from satellite images, acquired in the non-rainy season, correspond to the period when strong evaporation is noted and water shrinking. The exact boundaries are difficult to map with the high spatial resolution images.

\section{Discussion}

To construct indicators of the quality of the environment, the analysis of the 


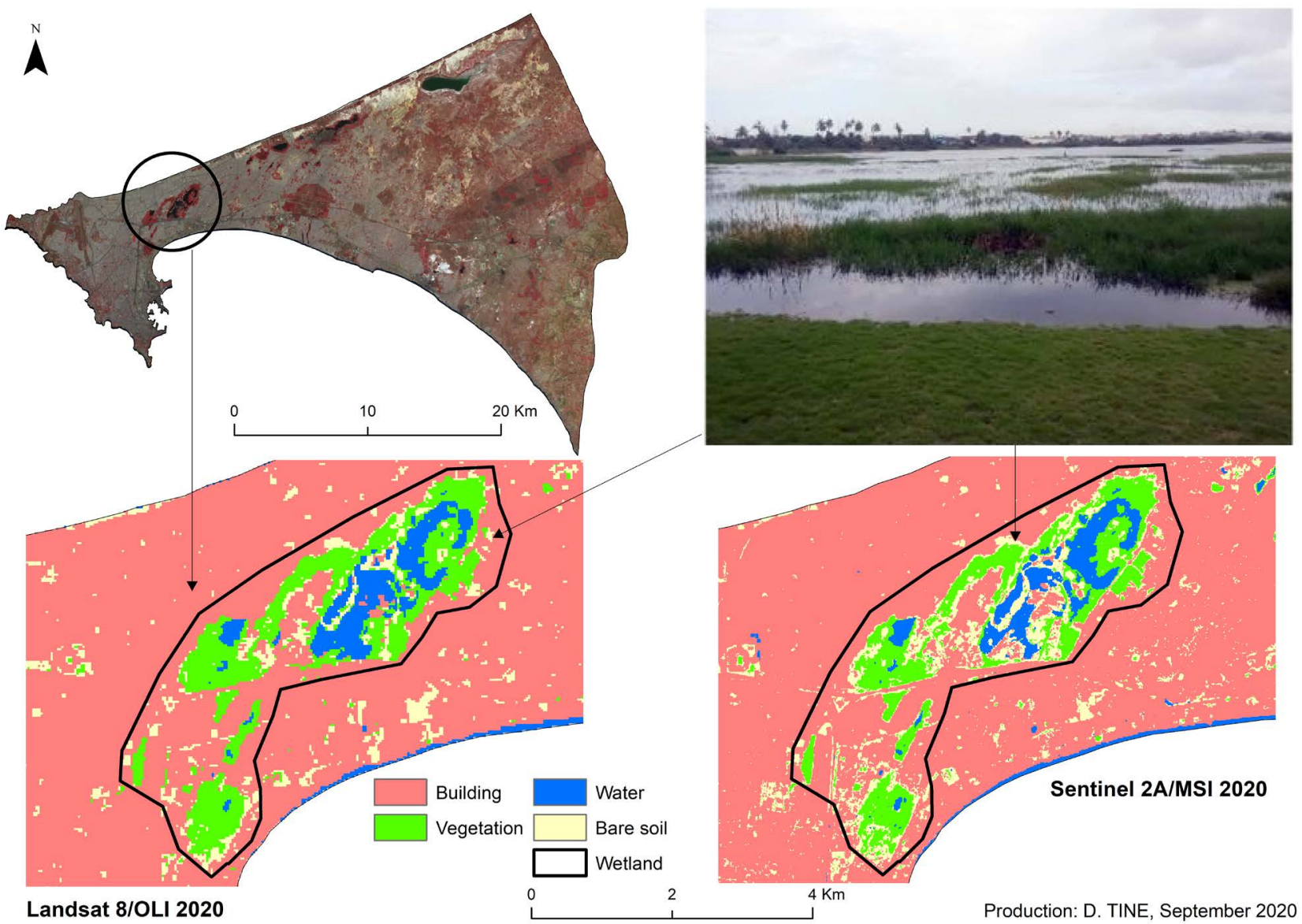

Figure 9. Mapping of Technopole wetlands through Landsat-8 OLI and Sentinel 2A.

morphology of urban vegetation seems essential. The contribution of the resolution appears mainly for the themes of urban vegetation [25]. The configuration of urban vegetation makes it difficult to identify and extract it from high spatial resolution satellite images. The density of the frame causes a pronounced mix of pixels and sometimes a shadow which is a hindrance for automatic processing because they mask certain areas and have a spectral response close to stagnant water [25]. This heterogeneous character of the pixels on the ground leads to an overestimation by place of the frame. An OLI pixel Landsat covers $900 \mathrm{~m}^{2}$ on the ground, a resolution much higher than residential plots. It is extremely difficult to find a pure pixel given these spatial characteristics of the OLI sensor. As for Sentinel-2 MSI, the fineness of the spatial resolution gives more details on the ground, which can impact the accuracy of the classifications. Within a Landsat- 8 OLI pixel, the HSR Sentienl-2A images, thanks to their spatial resolution, return homogeneous information on land use features. The same observations were made with vegetation indices (NDVI and SAVI). The results of the thresholding showed an underestimation of the vegetation cover. The minimum value of the vegetation indices is obtained near wetlands where there is strong leaf growth. Thresholding based on the minimum value may not take into account mixed pixels in which vegetation dominates. Their spectral signature looks like that of 
vegetation but different from that of pure pixels. In reality, it is difficult to qualify a sensor as ideal for mapping urban vegetation. However, this can have spatial and spectral characteristics suitable for the detection of leaf spaces in urban areas. The classification by SVM as well as the thresholding on the minimum value of the vegetation indices used gave satisfactory results despite the confusion due to the mix of pixels and the characteristics of the urban environment in Dakar. The data from the Landsat-8 OLI and Sentinel-2 sensors are suitable for the study of large-scale spatial phenomena but also for the mapping of somewhat specific spatial units in very heterogeneous environments. Analysis of the results obtained shows that very high resolution images with suitable processing methods can more accurately highlight urban vegetation.

\section{Conclusions}

The objective of this study is to evaluate the contribution of high spatial resolution images of Sentinel-2 and Landsat-8 OLI in the mapping and extraction of vegetation cover in urban areas. Two approaches are adopted: a supervised classification approach using the Support Vector Machine (SVM) algorithm and a thresholding approach based on the minimum value of the selected vegetation indices (NDVI and SAVI).

The results from the classifications are satisfactory with respective Kappa coefficients of $95 \%$ for Sentinel- 2 and $84 \%$ for Landsat- 8 OLI. These results, compared to the reality on the ground, make it possible to appreciate the contribution of spatial resolution in the extraction of urban vegetation. However, these results present imperfections which translate into confusion between certain classes such as buildings and bare soil. However, the results obtained by index thresholding were compared to the reality in the field and are considered acceptable, but the imperfections remain and can be explained by the complexity of the environment studied.

\section{Conflicts of Interest}

The authors declare no conflicts of interest regarding the publication of this paper.

\section{References}

[1] Nabucet, J. (2020) Contribution of Remote Sensing Data at Very High Spatial Resolution for the Mapping of Vegetation in Urban Areas. PhD Thesis, University of Brittany, Brittany, 341 p.

[2] Ballut, A. and Nguyen, J.P. (1984) Information Needs for Urban and Regional Planning: Contribution of Spatial Remote Sensing to the Satisfaction of These Needs. French Society of Photogrammetry and Remote Sensing, 93, 21-32.

[3] Forster, B.C. (1985) An Examination of Some Problems and Solution in Monitoring Urban Areas Satellite Platforms. International Journal of Remote Sensing, 6, 139-151. https://doi.org/10.1080/01431168508948430

[4] Welch, R. (1982) Spatial Resolution Requirement for Urban Studies. International 
Journal of Remote Sensing, 3, 138-146. https://doi.org/10.1080/01431168208948387

[5] Puissant, A. (2003) Geographic Information and Very High Resolution Images: Usefulness and Application in Urban Areas. Ph.D. Thesis, Luis Pasteur University Strasbourg I, Strasbourg, $166 \mathrm{p}$.

[6] Forster, B.C. (1982) Overcoming Urban Monitoring Problems with the New Generation Satellite Sensors. Proceedings of the International Symposium of Commission VII of the International Society for Photogrammetry and Remote Sensing, Toulouse, 13-17 September 1982, 889-896.

[7] IPCC (2007) Climate Change 2007: Synthesis Report. Intergovernmental Panel on Climate Change, Geneva, $114 \mathrm{p}$.

[8] IPCC (2014) Climate Change 2014: Impacts, Adaptation and Vulnerability. Intergovernmental Panel on Climate Change, Geneva, 40 p.

[9] Giguère, M. (2009) Urban Heat Island Control Measures, Literature Review. Quebec National Institute of Public Health, Quebec, 95 p.

[10] Pommier, G., Provendier, D., Gutleben, C. and Musy, M. (2014) Impact of Plants in the City. Research Program VegDUD, 61 p.

[11] Brunet, Y. (2017) Impact of Vegetation on Urban Microclimate and Air Quality. Chemistry and Big Cities, EDP Sciences, 272 p.

[12] Anquetil, V. (2010) Typology and Ecosystem Functions of Urban Vegetation: Methodological Contributions, Dissertation. National Institute of Horticulture and Landscape, Angers, 126p.

[13] World Health Statistics (WHO) (2018) Monitoring Health for the SDGs, Sustainable Development Goals. http://apps.who.int/iris/bitstream/handle/10665/272596/9789241565585-eng.pdf

[14] Ndiaye, M.L. (2014) Detection of Land Use Changes and Geomantic Modelling by Multi-Criteria Evaluation for the Mapping of Flood Vulnerable Areas in the Dakar. Senegal Region, Final Year Project Thesis, UM5/CRASTE-LF, 139 p.

[15] Michel, P. and Sall, M. (1984) Landscape Dynamics and Development of the Alluvial Valley of Senegal. In: Blanc-Pamard, C., Joël, B., Jean, B., Lassailly-Jacob, V., André, L., Eds., Le développement rural en questions. Paysages, espaces ruraux, systèmes agraires: Maghreb-Afrique noire-Mélanésie. ORSTOM, Paris, 89-109 (Mémoires ORSTOM; 106).

[16] Iovan, C., Boldo, D. and Cord, M. (2011) Modeling Vegetation in Urban Areas: Detection and Characterization from High Resolution Colour and Infrared Aerial Images. $10 \mathrm{p}$.

[17] Besse, M.-T. and Rouet, P. (2009) The Vegetation Cover of the Heart of the Paris Agglomeration: An Approach Using Precision Remote Sensing. CFC, 6 p.

[18] Rapinel, S., Clément, B. and Hubert-Moy, L. (2019) Wetland Mapping by Remote Sensing: A Multi-Scalar Approach for Environmental Planning. 21 p.

[19] Badiane, S.D. and Mbaye, E. (2018) Urban Wetlands with a Double Face in Dakar: Opportunity or Threat? $5 \mathrm{p}$.

[20] Samaaly, M. (1999) Study of Wetlands by Spatial Approach: Application to an Anthropogenic Marsh (Bourgneuf Marsh in Loire-Atlantique) and to a Natural Marsh (Kaw in Guyana). 149 p.

[21] Vacquié, L. and Thomas, H. (2012) Mapping Mountain Wetlands by Remote Sensing: Potentialities of Very High Spatial Resolution Images: Application to the Haut-Vicdessos valley (Pyrenees). 23 p.

[22] Charbonneau, L., Morin, D. and Brochu, R. (1985) Detection of Urban Land Use 
and Land Cover Units Using a SPOT Simulation. Quebec Geography Notebooks, 29, 29-47. https://doi.org/10.7202/021692ar

[23] Tidjani, A., Khorsi, A. and Ziani (2011) Detection of Vegetation from a Satellite Image. End of Studies Thesis, Amar Thelidji Laghouat University, Laghouat. https://www.memoireonline.com

[24] Be, A. (2016) Interests and Limitations of Remote Sensing for Crop Diagnosis. https://beapi.coop/l-essentiel-de-l-agriculture/interets-et-limites-de-la-teledetection -pour-diagnostiquer-les-cultures/\#: :text=Int\%C3\%A9r\%C3\%AAts\%20et\%20limite s\%20de\%20la\%20t\%C3\%A91\%C3\%A9d\%C3\%A9tection\%20pour\%20diagnostiquer \%20les\%20cultures,-Diagnostic\%20sol\&text=L'imagerie\%20a\%C3\%A9rienne\%20ap pliqu\%C3\%A9e\%20\%C3\%A0,1'\%C3\%A9chelle\%20intra\%2Dparcellaire

[25] Powerful, A. and Webber, C. (2001) The Use of High Resolution Images to Assess the Place of Vegetation in Urban Design. 10 p. 